\title{
A molecular roadmap for induced multi-lineage trans- differentiation of fibroblasts by chemical combinations
}

\begin{abstract}
Xiaoping Han ${ }^{1,2,10,11}$, Hao Yu ${ }^{3}$, Daosheng Huang ${ }^{1,11}$, Yang Xu ${ }^{1,11}$, Assieh Saadatpour ${ }^{4}$, Xia Li ${ }^{1,11}$, Lengmei Wang ${ }^{6}$, Jie $\mathrm{Yu}^{5}$, Luca Pinello ${ }^{4}$, Shujing Lai ${ }^{1,11}$, Mengmeng Jiang, ${ }^{1,11}$, Xueying Tian ${ }^{7}$, Fen Zhang ${ }^{1}$, Yanhong Cen ${ }^{1}$, Yuko Fujiwara $^{2}$, Wei Zhu', Bin Zhou ${ }^{7}$, Tianhua Zhou', Hongwei Ouyang ${ }^{1,10}$, Jianan Wang ${ }^{6,11}$, Guo-Cheng Yuan ${ }^{4}$, Shumin Duan ${ }^{3}$, Stuart H Orkin ${ }^{2,6}$, Guoji Guo ${ }^{1,2,10,11}$

${ }^{I}$ Center for Stem Cell and Regenerative Medicine, Zhejiang University School of Medicine, Hangzhou, Zhejiang 310058, China; ${ }^{2}$ Division of Pediatric Hematology/Oncology, Dana Farber Cancer Institute and Boston Children's Hospital, Harvard Medical School, Boston, MA 02115, USA; ${ }^{3}$ Institute of Neuroscience, Zhejiang University School of Medicine, Hangzhou, Zhejiang 310058, China; ${ }^{4}$ Department of Biostatistics and Computational Biology, Dana-Farber Cancer Institute, Harvard School of Public Health, Boston, MA 02115, USA; ${ }^{5}$ Department of Pathology, Zhejiang University School of Medicine, Hangzhou, Zhejiang 310058, China; ${ }^{6}$ The $2^{\text {nd }}$ Affiliated Hospital, Zhejiang University School of Medicine, Hangzhou, Zhejiang 310058, China; ${ }^{7}$ The State Key Laboratory of Cell Biology, Institute of Biochemistry and Cell Biology, Shanghai Institutes for Biological Sciences, Shanghai 200031, China; ${ }^{8}$ Howard Hughes Medical Institute, Boston, MA 02115, USA; ${ }^{9}$ Institute of Cell Biology, Zhejiang University School of Medicine, Hangzhou, Zhejiang 310058, China; ${ }^{10}$ Dr. Li Dak Sum \& Yip Yio Chin Center for Stem Cell and Regenerative Medicine, Zhejiang University, Hangzhou, Zhejiang 310058, China; ${ }^{11}$ Stem Cell Institute, Zhejiang University, Hangzhou, Zhejiang 310058, China
\end{abstract}

Cell Research (2017) 27:843. doi:10.1038/cr.2017.78; published online on 2 June 2017

Correction to: Cell Research (2017) 27:386-401. doi:10.1038/cr.2017.17; published online on 27 January 2017

During web production, there is an error in the web site for the labeling of Dr Bin Zhou, his affiliation is 7, not 8 . The pdf file is correct.

We apologize for any inconvenience that may have been caused by our error. 